\title{
Two-stage study of lung cancer risk modification by a functional variant in the 3'-untranslated region of SMAD5 based on the bone morphogenetic protein pathway
}

\author{
ZILI ZHANG $^{1 *}$, JIAN WANG $^{1,2^{*}}$, XIANSHENG ZENG $^{3 *}$, DEFU LI $^{1}$, MINGJING DING $^{4}$, RUIJUAN GUAN $^{1}$, \\ LIANG YUAN $^{1}$, QIPENG ZHOU ${ }^{1}$, MEIHUA GUO ${ }^{1}$, MINGMEI XIONG ${ }^{1}$, LIAN DONG ${ }^{1}$ and WENJU LU ${ }^{1,2,5}$
}

\begin{abstract}
${ }^{1}$ State Key Laboratory of Respiratory Diseases, Guangzhou Institute of Respiratory Disease, The First Affiliated Hospital, Guangzhou Medical University, Guangzhou, Guangdong 510120, P.R. China; ${ }^{2}$ Division of Translational and Regenerative Medicine, Department of Medicine, The University of Arizona, Tucson, AZ 85721-0202, USA; ${ }^{3}$ Department of Respiratory Medicine, Xiangyang Central Hospital, Xiangyang, Hubei 441021; ${ }^{4}$ Department of Respiratory Medicine, Inner Mongolia Autonomous Region People's Hospital, Hohhot 010017, Inner Mongolia Autonomous Region; ${ }^{5}$ Department of Laboratory

Medicine, The First Affiliated Hospital, Guangzhou Medical University, Guangzhou, Guangdong 510180, P.R. China
\end{abstract}

Received September 1, 2016; Accepted May 26, 2017

DOI: $10.3892 /$ mco.2017.1490

\begin{abstract}
Increasing evidence supports a key role for the bone morphogenetic protein $(B M P)$ signaling pathway in lung vasculogenesis and angiogenesis. Genetic variations in $B M P$ genes have been found to be correlated with cancer risk. In particular, the mutation in the 3'-untranslated region of BMPs may significantly affect gene function, leading to cancer susceptibility. The aim of the present study was to determine whether genetic variations in the components of the $B M P$ family are associated with lung cancer risk. A total of 314 tag single-nucleotide polymorphisms were identified in 18 genes, which are considered to either compose or regulate BMPs, and their association with lung cancer risk was evaluated in a two-stage case-control study with 4,680 cases and controls. A consistently significant association of SMAD5 rs12719482 with elevated lung cancer risk was observed in the three types of sources of populations (adjusted additive model in the combined population: Odds ratio $=1.32,95 \%$ confidence interval: $1.16-1.51)$. The lung cancer risk statistically significantly increased with the increasing number of variant alleles of SMAD5 rs12719482 in a dose-dependent pattern ( $\mathrm{P}$ for trend $=4.9 \times 10^{-5}$ ). Consistent evidence was identified for a significant interaction between the rs12719482 and cigarette smoking, performed as either a
\end{abstract}

Correspondence to: Professor Wenju Lu, State Key Laboratory of Respiratory Diseases, Guangzhou Institute of Respiratory Disease, The First Affiliated Hospital, Guangzhou Medical University, 151 Yanjiang Road, Guangzhou, Guangdong 510120, P.R. China E-mail:wlu92@yahoo.com

*Contributed equally

Key words: bone morphogenetic proteins, SMAD5, genetic variant, lung cancer, pathway continuous or discrete variable. These findings indicated that SMAD5 rs12719482 may be a possible candidate marker for susceptibility to lung cancer in the Chinese population.

\section{Introduction}

Lung cancer is one of the most common malignant tumors worldwide. Non-small-cell lung cancer (NSCLC) constitutes $\sim 80 \%$ of lung cancers, with a 5-year survival of only $15 \%$. Further study on the pathogenic mechanism of lung cancer is required to establish novel diagnostic or treatment strategies for this lethal disease.

Bone morphogenetic proteins (BMPs) belong to the transforming growth factor- $\beta$ (TGF- $\beta$ ) superfamily. Thus far, $>20$ BMPs have been identified in humans. Six of the type I and three of the type II serine/threonine kinase receptors have been shown to mediate $B M P$ signaling (1). Both type I and type II receptors consist of a $\mathrm{N}$-terminal extracellular ligand-binding domain and a C-terminal serine/threonine kinase domain (2). When the BMP ligand binds to preformed hetero-oligomeric complexes, the SMAD-dependent pathway may be activated (3). The pathway-restricted SMADs (such as SMAD1, 2, 3, 5 or 8) are recruited and translocated into the nucleus with the assistance of SMAD4 and regulate the transcription of target genes, referred to as the SMAD-dependent pathway. The activated $B M P$ signaling may then enhance invasion and bone metastasis of cancer cells via the $S M A D$ pathway (4). BMPs significantly affect embryonic and postnatal development and maintenance of homeostasis in organs and tissues; they are also associated with cell proliferation, differentiation, motility and survival (5) and they are involved in the development and progression of certain malignant tumors, such as lung, prostate and breast cancer (6). Recently, accumulating evidence demonstrated that BMPs also participate in tumor-related angiogenesis $(7,8)$.

It was hypothesized that the polymorphisms in the $B M P$ component and regulatory genes may contribute to the 
genetic susceptibility to lung cancer. To test this hypothesis, a two-stage case-control study was performed, including a total of 2,340 patients and 2,340 cancer-free controls, with the specific aim of evaluating the effects of gene polymorphisms in the 18 selected genes related to the $B M P$ pathway $[B M P 2$, 4, 6, 7 and 9,SMAD1, 4, 5,6,7 and 8,SMURF1 and 2,ACTR2, ALK2 (ACVR1), ALK3 (BMPR-1A), ALK6 (BMPR-1B) and $B M P R 2]$ on lung cancer. To the best of our knowledge, this is the first study to evaluate the associations between a comprehensive panel of genetic variants related to the $B M P$ pathway and lung cancer, and to identify certain patient subgroups that may be at high risk of developing lung cancer.

\section{Patients and methods}

Study population and design. The study design and subject recruitment have been previously described (9). Briefly, two independent case-control projects were conducted. The 'stage I' setting (discovery) included 1,422 patients and 1,422 controls mainly from Guangzhou, Guangdong, China (9). The 'stage II' setting (replication) included 918 cases and 918 controls from Xianyang, Hubei, China, to test the results of stage I. A total of 2,340 cases with histopathologically diagnosed lung cancer were enrolled from September, 2009 onwards. The 2,340 cancer-free controls were recruited during the same time period. The characteristics of the cases and controls are summarized in Table I. Structured questionnaires were conducted by experienced interviewers using a standardized protocol. The inclusive criteria and definition of cigarette smoking were previously detailed (9). This project was approved by the Institutional Ethics Committee of each participating center. All the participants provided written informed consent prior to enrolment.

Blood sampling, SNP selection and genotyping. Blood samples were provided by all the participants. The genomic DNA was extracted with the Qiagen Blood DNA kit (Qiagen, Valencia, CA, USA). A total of 18 genes associated with the BMP pathway were selected. For each of those genes, the tagSNPs were selected using Haploview 4.2 software (http://haploview. software.informer.com/4.2/). The genotypes of 314 selected tagSNPs and their associations with lung cancer are detailed in Fig. $1\left(\mathrm{P}<1.6 \times 10^{-4}\right)$ and Table II $(\mathrm{P}<0.05)$. The Illumina high-throughput genotyping platform and the Illumina Beadstudio software were used (Illumina, San Diego, CA, USA). To ensure quality control, genotyping was performed using blinded methods and the analysis was conducted separately by two researchers. Over $15 \%$ of the samples were used for confirmation, and the outcomes were $100 \%$ concordant. The genotyping call rates were all $>95 \%$.

Statistical analysis. The $\chi^{2}$ test was used to evaluate the qualitative data and the Hardy-Weinberg equilibrium (HWE) applied. The Akaike Information Criteria (AIC) (10) were employed to select a genetic model for each SNP. Odds ratios (ORs) and corresponding $95 \%$ confidence intervals (CIs) were calculated by an unconditional logistic regression model adjusted for confounding factors. Stratification analyses were performed with the variables of interest. The pairwise linkage disequilibrium among the SNPs and haplotype blocks/frequencies were measured accordingly (11-13). Homogeneity between stage I and II populations was assessed by the Breslow-Day test. Statistical power was assessed by Quanto 1.2. software (http://biostats.usc.edu/Quanto.html) and corrected by the Bonferroni test for each of the 314 SNPs at a significance level of $0.05 / 314=1.6 \times 10^{-4}$ (Fig. 1). All the analyses were performed using SAS 9.2 software (SAS Institute Inc., NC, USA).

\section{Results}

Characteristics of the populations. As summarized in Table I, consistent results were found in stages I and II, with significant differences in smoking status, pack-years and gender-smoking $(\mathrm{P}<0.001$ for all); there was no statistically significant deviation in the distributions of gender and age between the case and control groups ( $\mathrm{P}>0.05$ for all). The frequency distribution of smoking status was heterogeneous (Breslow-Day test $\mathrm{P}<0.0001)$. The two groups were then combined to increase the study power, and almost identical change tendencies were identified separately for stages I and II.

Genetic variants in SMAD5 and lung cancer risk. As mentioned above, 314 tagSNPs were selected from 18 genes associated with the $B M P$ pathway; those with $\mathrm{P}<0.05$ in stage I, stage II, or the combined populations, are shown in Table II. Among these tagSNPs, consistently significant associations were found between SMAD5 rs12719482 and lung cancer risk in the three types of sources of populations $(\mathrm{P}<0.05$ for all). In particular, when combining stages I and II, the differences were more significant compared with any individual stage in the additive genetic model $\left(\mathrm{P}=4.39 \times 10^{-5}\right)$. All observed genotype distributions among these groups were in HWE ( $\mathrm{P} \geq 0.05$ for all). The frequency distributions of the genotypes of rs12719482 are also summarized in Table III. In the stage I set, significant associations were found between rs12719482 T>C genotypes, as well as alleles, and lung cancer risk. Compared with zero-risk genotype carriers, the one- or two-risk genotype was associated with increased lung cancer risk in a dose-dependent manner (adjusted $\mathrm{OR}=1.27$, 95\% CI: 1.07-1.50, $\mathrm{P}=0.0060$ ), while the $\mathrm{rs} 12719482 \mathrm{CT} / \mathrm{CC}$ genotype was associated with a significantly increased risk in the dominant genetic model $(\mathrm{OR}=1.26$; 95\% CI: $1.05-1.52$; $\mathrm{P}=0.0133$ ). Allele $\mathrm{C}$ increased the risk of lung cancer by $23 \%$ compared with the wild-type T. Concordant with the results of rs12719482 T>C and lung cancer risk analysis in stage I, the variant genotype analyses revealed almost similar change tendencies in the stage II setting. Importantly, the association remained statistically significant when all the participants were combined.

Stratification analysis of $r$ s $12719482 T>C$ in the combined study. The associations of the rs12719482 (T>C) with lung cancer risk stratified by selected variables using the additive model was then assessed according to the AIC pattern. As shown in Table IV, individuals exhibited a significantly increased lung cancer risk as the number of variant alleles increased, in the ages of $\leq 60$ as well as $>60$ years $(\mathrm{P}=0.0039$ and $\mathrm{P}=0.0087$, respectively), in both genders $(\mathrm{P}=0.0155$ for men and $\mathrm{P}=0.0006$ for women), in smokers as well as non-smokers $(\mathrm{P}=0.0135$ and $\mathrm{P}=0.0012$, respectively), in $\geq 20$ and 0 pack-years $(\mathrm{P}=0.0407$ 


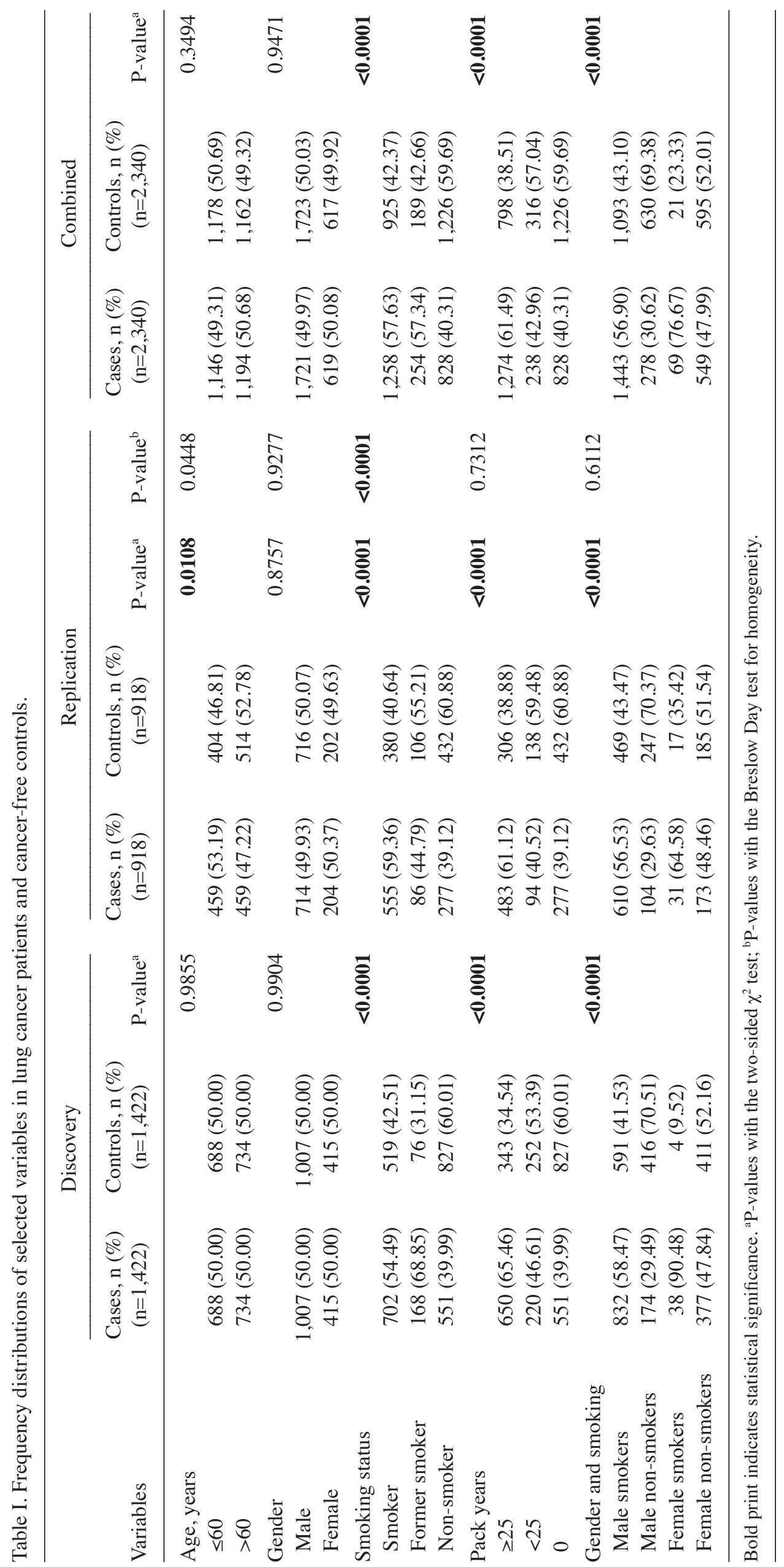


A

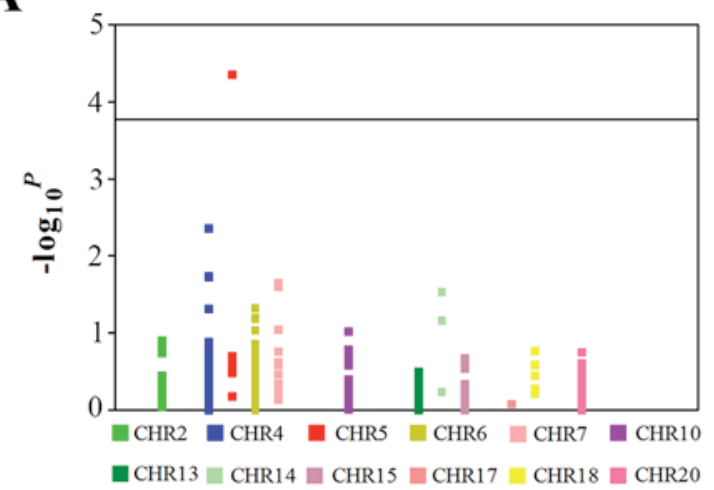

C

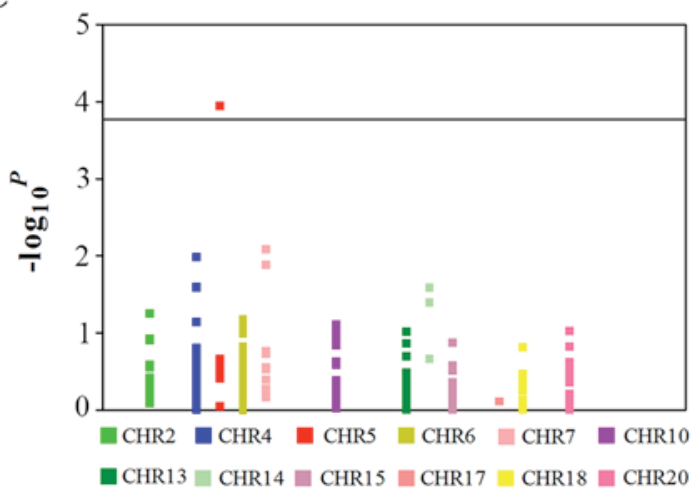

B

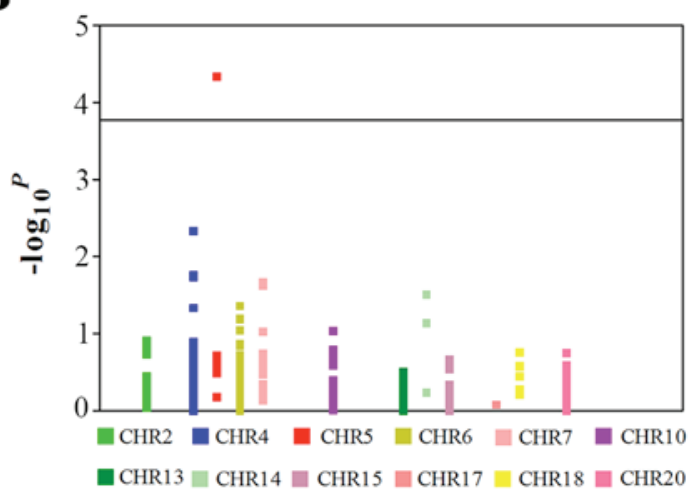

D

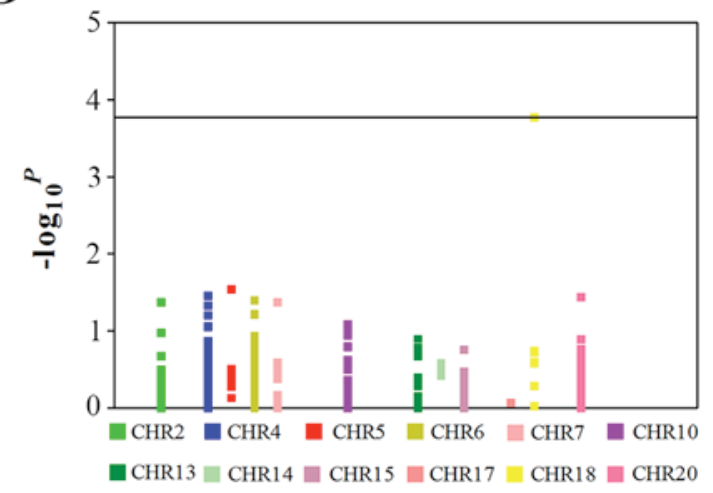

Figure 1. Results on the association of 314 tagSNPs with lung cancer in the Han Chinese population. (A) Allele genetic model; (B) additive genetic model; (C) dominant genetic model; and (D) recessive genetic model. The Bonferroni significant threshold was $1.6 \times 10^{-4}$. SNP, single-nucleotide polymorphism; CHR, chromosome.

and $\mathrm{P}=0.0017$, respectively) and in both male smokers and female non-smokers $\left(\mathrm{P}_{\max }=0.0350\right)$ following adjustment for confounding factors. There were also statistically significant multiplicative interactions among gender, smoking status, pack-years and allele genes $(\mathrm{P}<0.0001$ for all).

\section{Discussion}

Lung cancer remains a major health concern, due to its 5-year survival of only $15 \%$ and associated high medical costs. Genetic biomarkers may help identify susceptible subgroups for screening, diagnosis and even therapy in earlier stages, and it may also be beneficial for clinical outcomes. A total of $314 \mathrm{tag}$ SNPs were identified in the 18 pivotal genes from the $B M P$ pathway and evaluated for their association with lung cancer risk. In the present study, a significant role was indicated for SMAD5 in TGF- $\beta$-mediated lung cancer. The findings were concordant for a significant association between the SMAD5 rs12719482 and cigarette smoking. In addition, using Function Prediction websites (https://snpinfo.niehs.nih. gov/snpinfo/snpfunc.html), it was demonstrated that SMAD5 rs12719482 may be a susceptibility marker by decreasing the expression of SMAD5 through binding of one of hsa-miR-1270, hsa-miR-571 or hsa-miR-920 to the polymorphic site in the Chinese population. To the best of our knowledge, the present study is the first to estimate the associations between a comprehensive panel of genetic polymorphism of BMP genes and lung cancer risk, and to investigate the potential susceptible subgroups. A series of functional experiences are required in the future, as this is the first epidemiological study supporting an association between SMAD5 rs12719482 and lung cancer risk.

Identification of SMAD proteins has been helpful in promoting the understanding of $T G F-\beta$ signaling. BMPs may signal through both canonical and non-canonical pathways. In particular, in the canonical pathway, the BMP functions through BMP ligand (BMPR1)-binding membrane-bound receptors, resulting in the phosphorylation of intracellular mediators, known as the receptor-regulated SMADs (R-SMADs). SMADs are crucial intracellular signaling transmitters of the TGF- $\beta$ superfamily of peptide growth factors that regulate a series of biological processes (5). Controlling SMAD activity and protein levels are crucial for proper signaling by TGF- $\beta$ and its related factors. Due to their ubiquitous expression and various functions as regulators in various organs of the body, BMPs were referred to as body morphogenetic proteins (14), as disruptions of BMP signaling are associated with a wide variety of defects or severe pathologies, such as vascular diseases, skeletal diseases and cancer.

$S M A D 5$, one of the $M A D$-homologues, is situated on chromosome $5 \mathrm{q} 31$ in humans and acts downstream of the TGF- $\beta$ receptors. It is an important component of R-SMADs (SMAD1/5/8) and the closest homolog of SMAD1, which plays a role in the $B M P-2$ signaling pathway (15). Accumulating evidence indicates that the SMAD5 gene functions in the signaling pathway involving the inhibitory effect of TGF- $\beta$ on 


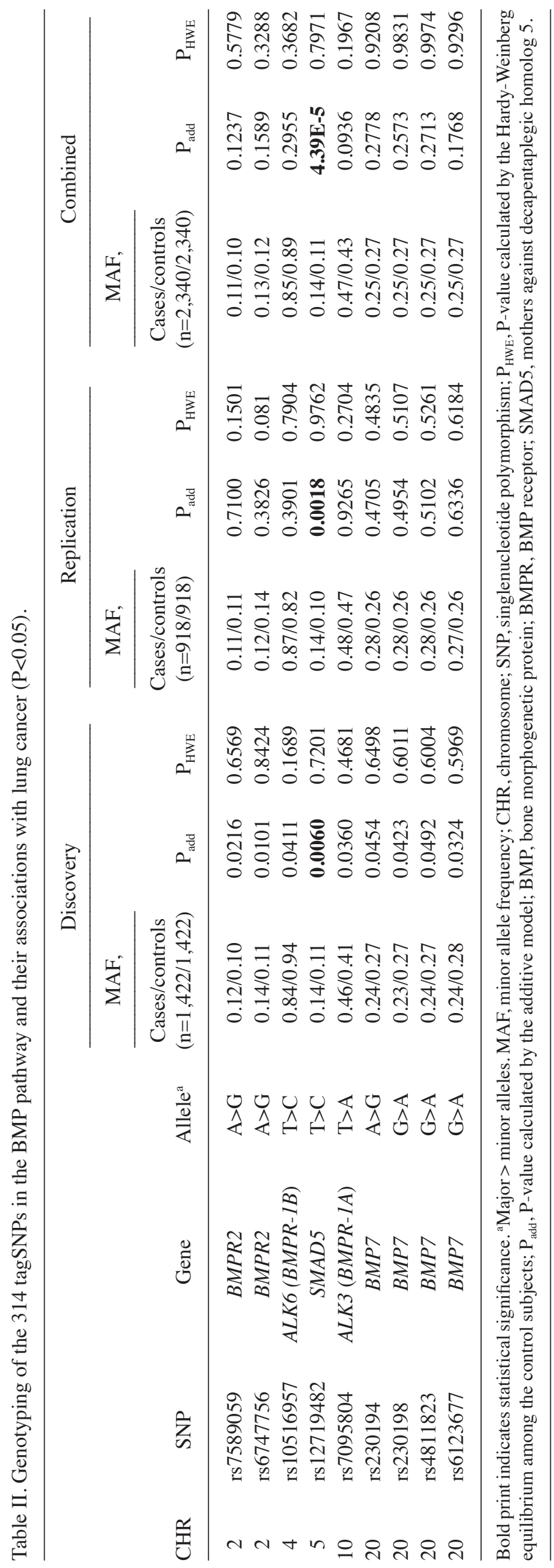




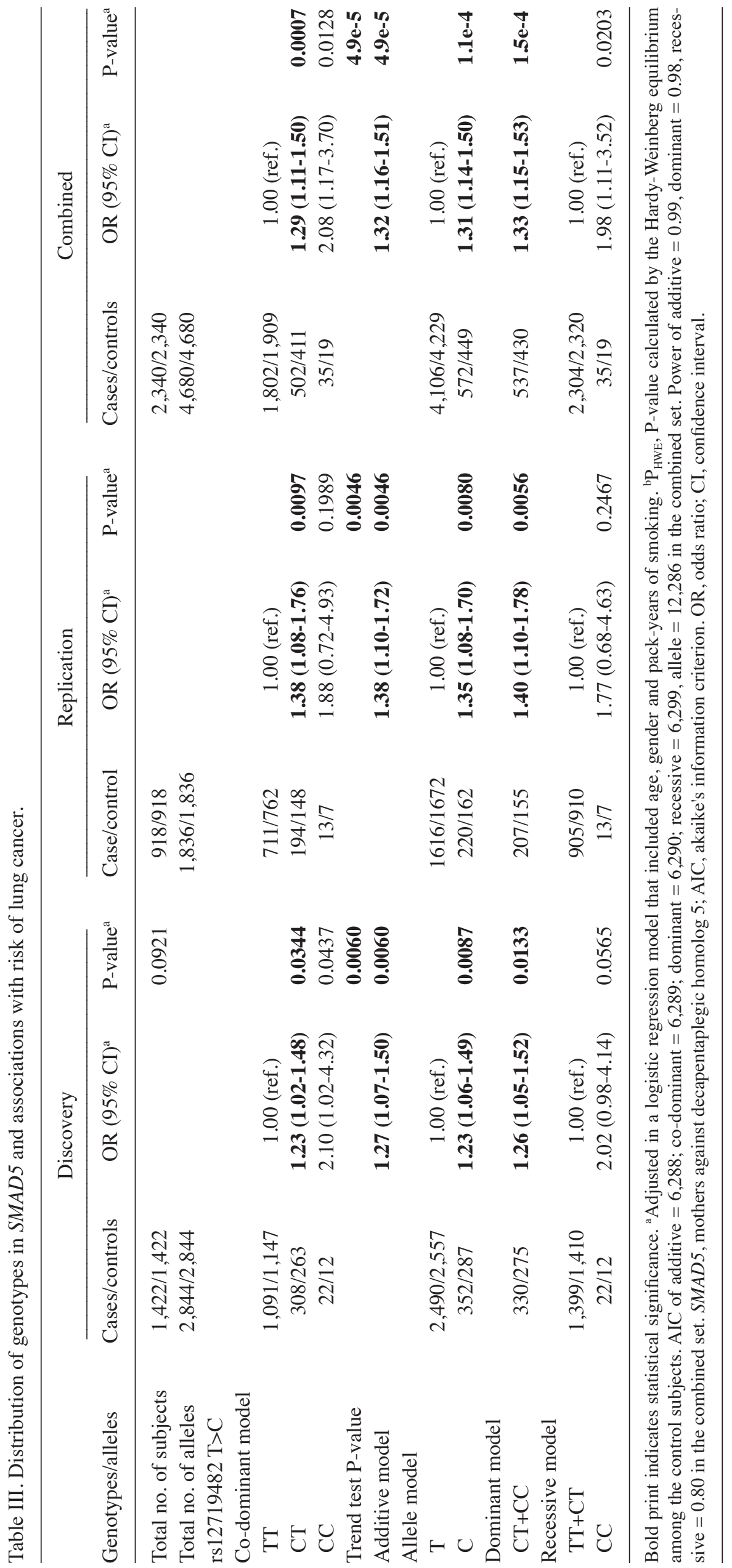




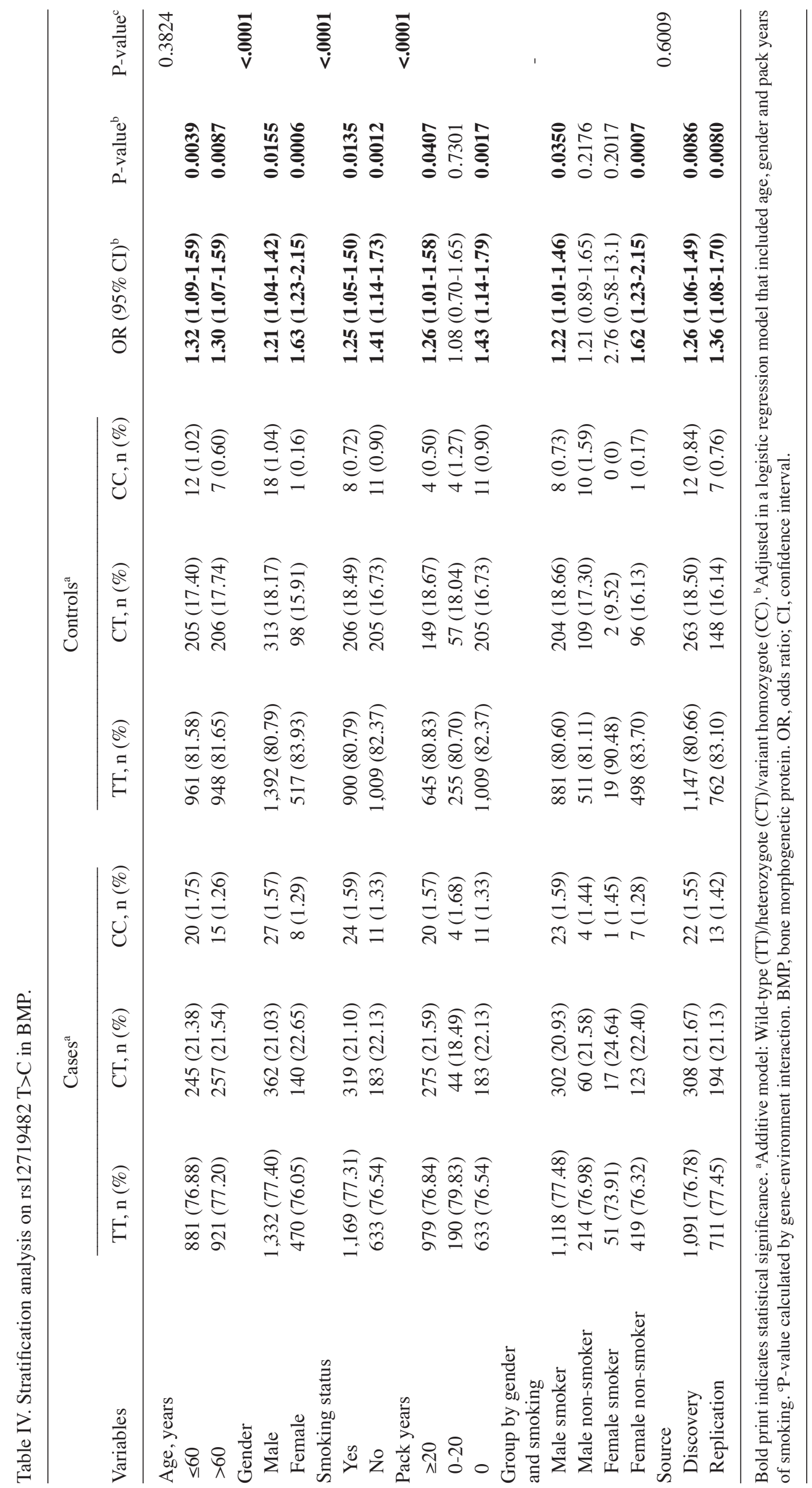


cancer. For example, SMAD5 was considered to be a candidate tumor suppressor gene in myelodysplastic syndrome and acute myeloid leukemia and is found in human hematopoietic progenitor cells.

Animal studies have also revealed specific functions of SMAD5. In the absence of SMAD5, mice die between embryonic day (E)9.5 and E10.5 from impaired circulation. Difficulties in angiogenesis and diminished number of vascular smooth muscle cells (VSMCs) have been reported in SMAD5-deficient mice $(16,17)$. Mice lacking SMAD5 exhibited increased apoptosis of cardiac myocytes and craniofacial abnormalities. At the cellular level, previous studies demonstrated that conditional knockout of SMADI and SMAD5 in granulosa cells results in the formation of vascularized granulosa cell tumors with complete penetrance and with an elevated incidence of peritoneal metastases and hemorrhagic ascites with increasing age (18). In addition, SMAD5-deficient embryos exhibited defects in angiogenesis leading to decreased VSMC numbers and enlarged dilated vessels. A disorganized allantois was formed, primordial germ cells were largely diminished, with left-right asymmetry and impaired embryo turning, all resulting from SMAD5 deficiency $(19,20)$. Furthermore, the $S M A D 5^{-/-}$yolk sac may lead to abnormalities in the vitelline network (17). However, the potential molecular mechanism underlying SMAD5-dependent function remains to be further elucidated.

The results of the present study also indicated that the effect of SMAD5 rs12719482 (T>C) on humans was detrimental, particularly among heavy smokers and when compared between non-smokers and light smokers. In particular, the effect of SMAD5 rs12719482 (T>C) was more prominent among light smokers (0 pack-years) and non-smokers compared with the wild-types TT. For example, the heavy smoking group ( $\geq 20$ pack-years) exhibited a 1.26 -fold increased risk for rs12719482 (T>C), whereas its effect was 1.43 among light smokers when wild-type TT was used as the reference genotype in the additive model. When stratified by smoking status, non-smokers showed a 1.41-fold increased risk for rs12719482 ( $>C$ ) compared with the TT genotype. Therefore, there may be a modified association between cigarette smoking and the genotype variants of SMAD5 rs12719482 (T>C). The potential mechanism underlying the interaction of SMAD5 and smoking is not clear. However, the role of SMAD5 rs12719482 (T>C) was highlighted among non-smokers and light smokers. Therefore, additional evidence and functional studies are required. In addition, in the present study the frequency distribution of smoking status was not homogeneous between cases and controls in stage I as well as stage II, reflecting the differences in lifestyle.

Several inherent biases of this study should be elucidated. First, patients were mainly selected from hospitals and the controls were selected from the center of health examination during a routine health check; therefore, there was an inherent selection bias. However, by matching the controls to the patients according to age, gender and residential area, the underlying confounding factors may be minimized. Second, risk factors other than smoking status, such as occupational exposure and nutrition, which may interact with $B M P$ genotypes or function as potential confounding factors, were not included in our study. Possible interactions between those factors should be thoroughly investigated in future studies. Finally, the functional relevance of rs12719482 remains unknown, and further related investigations should be performed.

In conclusion, we herein provided evidence indicating that the SMAD5 rs12719482 polymorphism and its interactions with smoking status may be a potential etiology of lung cancer in Chinese patients. These findings remain to be tested by larger scale studies in different ethnic groups.

\section{Acknowledgements}

The present study was supported by grants from the National Natural Science Foundation of China (81520108001, 81220108001 and 81170052), the 973 Key Scheme of China (2015CB553406), the National Key Research and Development Project (2016YFC0903700), the Guangdong Province Universities and Colleges Pearl River Scholar Funded Scheme (2014, to W. Lu), the Guangdong Province Universities and Colleges Key Grant for Innovative Research (cxzd1142), the Guangzhou Municipal Research Project (201607020030), the Guangzhou Department of Education Innovative Team (13C08), the Guangzhou Department of Education Yangcheng Scholarship (12A001S), the Guangdong Natural Science Foundation (2016A030313593) and the Guangzhou Municipal University Research Projects (1201430298).

\section{References}

1. Babitt JL, Huang FW, Wrighting DM, Xia Y, Sidis Y, Samad TA, Campagna JA, Chung RT, Schneyer AL, Woolf CJ, et al: Bone morphogenetic protein signaling by hemojuvelin regulates hepcidin expression. Nat Genet 38: 531-539, 2006.

2. Yu PB, Beppu H, Kawai N, Li E and Bloch KD: Bone morphogenetic protein (BMP) type II receptor deletion reveals BMP ligand-specific gain of signaling in pulmonary artery smooth muscle cells. J Biol Chem 280: 24443-24450, 2005.

3. Daly AC, Randall RA and Hill CS: Transforming growth factor beta-induced Smad1/5 phosphorylation in epithelial cells is mediated by novel receptor complexes and is essential for anchorage-independent growth. Mol Cell Biol 28: 6889-6902, 2008.

4. Katsuno Y, Hanyu A, Kanda H, Ishikawa Y, Akiyama F, Iwase T, Ogata E, Ehata S, Miyazono K and Imamura T: Bone morphogenetic protein signaling enhances invasion and bone metastasis of breast cancer cells through Smad pathway. Oncogene 27: 6322-6333, 2008.

5. Kang JS, Saunier EF, Akhurst RJ and Derynck R: The type I TGF-beta receptor is covalently modified and regulated by sumoylation. Nat Cell Biol 10: 654-664, 2008.

6. Cheng H, Jiang W, Phillips FM, Haydon RC, Peng Y, Zhou L, Luu HH, An N, Breyer B, Vanichakarn P, et al: Osteogenic activity of the fourteen types of human bone morphogenetic proteins (BMPs). J Bone Joint Surg Am 85-A: 1544-1552, 2003.

7. Zhang L, Ye Y, Long X, Xiao P, Ren X and Yu J: BMP signaling and its paradoxical effects in tumorigenesis and dissemination. Oncotarget 7: 78206-78218, 2016.

8. Ye L and Jiang WG: Bone morphogenetic proteins in tumour associated angiogenesis and implication in cancer therapies. Cancer Lett 380: 586-597, 2015.

9. Zhang Z, Wang J, He J, Zheng Z, Zeng X, Zhang C, Ye J, Zhang Y, Zhong $\mathrm{N}$ and Lu W: Genetic variants in MUC4 gene are associated with lung cancer risk in a Chinese population. PLoS One 8: e77723, 2013

10. Akaike H: A new look at the statistical model identification. IEEE Trans Automat C ontr AC 19: 716-723, 1974.

11. Lewontin RC: On measures of gametic disequilibrium. Genetics 120: 849-852, 1988

12. Gabriel SB, Schaffner SF, Nguyen H, Moore JM, Roy J, Blumenstiel B, Higgins J, DeFelice M, Lochner A, Faggart M, et al: The structure of haplotype blocks in the human genome. Science 296: 2225-2229, 2002. 
13. Stephens M and Donnelly P: A comparison of bayesian methods for haplotype reconstruction from population genotype data. Am J Hum Genet 73: 1162-1169, 2003.

14. Wagner DO, Sieber C, Bhushan R, Börgermann JH, Graf D and Knaus P: BMPs: From bone to body morphogenetic proteins. Sci Signal 3: mrl, 2010.

15. Hoodless PA, Haerry T, Abdollah S, Stapleton M, O'Connor MB, Attisano L and Wrana JL: MADR1, a MAD-related protein that functions in BMP2 signaling pathways. Cell 85: 489-500, 1996.

16. Chang H, Huylebroeck D, Verschueren K, Guo Q, Matzuk MM and Zwijsen A: Smad5 knockout mice die at mid-gestation due to multiple embryonic and extraembryonic defects. Development 126: 1631-1642, 1999.

17. Yang X, Castilla LH, Xu X, Li C, Gotay J, Weinstein M, Liu PP and Deng CX: Angiogenesis defects and mesenchymal apoptosis in mice lacking SMAD5. Development 126: 1571-1580, 1999.
18. Pangas SA, Li X, Umans L, Zwijsen A, Huylebroeck D, Gutierrez C, Wang D, Martin JF, Jamin SP, Behringer RR, et al: Conditional deletion of Smad1 and Smad5 in somatic cells of male and female gonads leads to metastatic tumor development in mice. Mol Cell Biol 28: 248-257, 2008.

19. Chang H and Matzuk MM: Smad5 is required for mouse primordial germ cell development. Mech Dev 104: 61-67, 2001.

20. Chang H, Zwijsen A, Vogel H, Huylebroeck D and Matzuk MM: Smad5 is essential for left-right asymmetry in mice. Dev Biol 219: 71-78, 2000.

(i) (9) This work is licensed under a Creative Commons Attribution-NonCommercial-NoDerivatives 4.0 International (CC BY-NC-ND 4.0) License. 